\title{
Loss of CDX2 gene expression is associated with DNA repair proteins and is a crucial member of the Wnt signaling pathway in liver metastasis of colorectal cancer
}

\author{
CSABA TÓTH ${ }^{1,5}$, FARKAS SÜKÖSD ${ }^{2}$, ERZSÉBET VALICSEK ${ }^{3}$, ESTHER HERPEL ${ }^{1,4}$, \\ PETER SCHIRMACHER ${ }^{1}$ and LÁSZLÓ TISZLAVICZ ${ }^{2}$ \\ ${ }^{1}$ Institute of Pathology, University Hospital Heidelberg, D-69120 Heidelberg, Germany; \\ Departments of ${ }^{2}$ Pathology and ${ }^{3}$ Oncotherapy, University of Szeged, 6725 Szeged, Hungary; \\ ${ }^{4}$ Tissue Bank of The National Center for Tumor Diseases (NCT), D-69120 Heidelberg, Germany
}

Received July 30, 2017; Accepted December 13, 2017

DOI: $10.3892 / \mathrm{ol} .2018 .7756$

\begin{abstract}
Caudal type homeobox 2 (CDX2) has been well-established as a diagnostic marker for colorectal cancer (CRC); however, less is known about its regulation, particularly its potential interactions with the DNA repair proteins, adenomatous polyposis coli (APC) and $\beta$-catenin, in a non-transcriptional manner. In the present study, the protein expression of CDX2 was analyzed, depending on the expression of the DNA repair proteins, mismatch repair (MMR), O6-methylguanine DNA methyltransferase (MGMT) and excision repair cross-complementing 1 (ERCC1), and its importance in Wnt signaling was also determined. A total of 101 liver metastases were punched into tissue microarray (TMA) blocks and serial sections were cut for immunohistochemistry. For each protein, an immunoreactive score was generated according to literature data and the scores were fitted to TMA. Subsequently, statistical analysis was performed to compare the levels of expression with each other and with clinical data. CDX2 loss of expression was observed in $38.5 \%$ of the CRC liver metastasis cases. A statistically significant association between CDX2 and each of the investigated MMRs was observed: MutL Homolog $1(\mathrm{P}<0.01)$, MutS protein Homolog (MSH) 2 ( $\mathrm{P}<0.01)$, MSH6 $(\mathrm{P}<0.01)$, and postmeiotic segregation increased $2(\mathrm{P}=0.040)$. Furthermore, loss of MGMT and ERCC1 was also associated with CDX2 loss $(\mathrm{P}=0.039$ and $\mathrm{P}<0.01$, respectively). In addition, $\mathrm{CDX} 2$ and ERCC1 were inversely associated with metastatic tumor size $(\mathrm{P}=0.038$ and
\end{abstract}

Correspondence to: Dr Csaba Tóth, Institute of Pathology, University Hospital Heidelberg, Im Neuenheimer Feld 224, D-69120 Heidelberg, Germany

E-mail: c.toth@patho-trier.de; csaba.toth@med.uni-heidelberg.de

Present address: ${ }^{5} \mathrm{MVZ}$ for Histology, Cytology and Molecular Diagnostics Trier, D-54296 Trier, Germany

Key words: caudal type homeobox 2, colorectal cancer, liver metastasis, DNA repair proteins
$\mathrm{P}=0.027$, respectively). Sustained CDX2 expression was associated with a higher expression of cytoplasmic/membranous $\beta$-catenin and with nuclear APC expression $(\mathrm{P}=0.042$ and $\mathrm{P}<0.01$, respectively). In conclusion, CDX2 loss of expression was not a rare event in liver metastasis of CRC and the results suggested that CDX2 may be involved in mechanisms resulting in the loss of DNA repair protein expression, and in turn methylation; however, its exact function in this context remains to be elucidated.

\section{Introduction}

Colorectal cancer (CRC) is the third most deadly cancer worldwide accounting for more than 600,000 deaths annually (1). At the diagnosis, a quarter of the patients with primary CRC have synchronous hepatic metastasis, and more than $50 \%$ of the patients with CRC will develop liver metastases in the course. Almost half of the patients undergoing resection for primary CRC eventually develop metachronous liver metastasis. Survival in metastatic cases is rarely longer than three years (2). Interestingly, although caudal type homeobox 2 (CDX2) is widely used in the daily routine diagnostic, there are less than sixty publications in the last sixty years performed on human tissue investigating the role of CDX2 (3).

The Cdx family of transcription factors contributes also to the CRC phenotype, but a mechanism by which CDX2 expression is lost or downregulated in colorectal tumors is currently not clear. The CDX2 is necessary for the proper development of the intestinal tract and is crucial for development and homeostasis of the intestinal epithelium throughout life (1). The role of $\mathrm{Cdx} 2$ in colorectal carcinogenesis is multi-sided. The CDX2 expression is reduced in CRC and its expression is inversely correlated to tumor grade, tumor stage and lymph node metastasis (4). Loss of CDX2 expression can strongly predict high level $\mathrm{CpG}$ island methylation phenotype (CIMP-H) independently from microsatellite status of CRCs. Thus Cdx2 was proposed as a surrogate marker for CIMP-H (5). In addition, CDX2 was attributed to play a regulatory role in apoptosis and DNA repair. Colon epithelium with decreased CDX2 expression lead to impaired apoptosis potential after $\gamma$-irradiation, 
thus resulting in higher resistance to genotoxic stress. Besides, the effect of CDX2 in DNA repair activity can contribute to its attributed tumor suppressor function (6).

DNA methylation of tumor suppressor genes resulting in its transcriptional inactivation and has been identified as an important mechanism. CIMP characterized by the extensive hypermethylation of multiple $\mathrm{CpG}$ islands, and belongs to one of the major mechanisms in the colorectal carcinogenesis (7). O6-methylguanine DNA methyltransferase (MGMT), a surrogate marker for CIMP, gene promoter methylation plays an important role in colorectal carcinogenesis. Loss of MGMT expression, which is secondary to gene promoter methylation, occurs in approximately $30-40 \%$ of metastatic CRC. In addition, loss of MGMT expression results in high response to alkylating agents (i.e., dacarbazine or temozolomide) (8). Thus, MGMT is believed to have predictive potential for therapy.

A further level of DNA damage defence mechanism is represented by the mismatch repair (MMR) system, which take part not only in the DNA repair processes, but also in the regulation of cell cycle check-points and apoptosis (9). Deficiency of MMR proteins (i.e., MLH1 and MSH2) is responsible for resistance to various chemotherapeutic drugs and subsequently for resistance to apoptosis (9). Interestingly, loss of MGMT expression is more frequent in CRC with microsatellite instability, suggesting that methylated MGMT selects cellular clones with MMR deficient status (8). Moreover, MMR deficiency is also correlated with loss of CDX2 (10).

Excision repair cross-complementing 1 (ERCC1) is a structure specific DNA repair endonuclease responsible for 5 ' incision (5'-endonuclease), a key enzyme in nucleotide excision repair (NER) pathway and is essential for repair of platinum-DNA adducts, thus associated with therapy resistance to platinum-containing compounds (i.e., cisplatin) $(11,12)$.

Aberrant $\beta$-catenin expression and disturbed Wnt signaling is recognized as an important event in the genesis of several malignancies, especially in CRC. $\beta$-catenin mutations or loss-of-function mutations of the adenomatous polyposis coli (APC) tumor suppressor gene appear to be crucial steps in the progression of this disease (13). APC and $\beta$-catenin were found to traffic independently from each other into and out of the nucleus in response to internal and external signals. This fact has prompted debate about the previously proposed role of APC as a $\beta$-catenin chaperone (14). Germline mutations in the APC gene cause familial adenomatous polyposis (FAP), and over $80 \%$ of CRCs (both inherited and sporadic) carry truncating mutations that inactivate the APC protein. Most of these mutations occur in the so-called 'mutation cluster region' of the APC gene, accounting for a truncated protein incapable of binding regulatory proteins (i.e., Axin) or associating with microtubules. The relevance of truncating mutations for $\beta$-catenin is enormous: Mutated APC cannot stimulate its degradation (because of its failure to bind Axin), although APC still can bind to $\beta$-catenin (albeit less efficiently) $(14,15)$. $\beta$-catenin has been observed to accumulate in the nuclei of colon cancer cells, which results from the inability of APC to promote $\beta$-catenin degradation, rather than a lack of export function, leading to nuclear accumulation of $\beta$-catenin in APC-mutant tumor cells (14). There are only few studies that focused on interactions between CDX2 and Wnt signalling in colon cancer. It has been demonstrated that CDX2 can inhibit the transcriptional activity of $\beta$-catenin/TCF lines in a non-transcriptional way (4).

Expression of CDX2 in association with DNA repair proteins and members of Wnt signaling pathway has not been studied previously in liver metastasis of CRC. In this study, we analysed the expression distribution of CDX2 in matters of expression status of DNA repair proteins (MMR proteins, MGMT and ERCC1), APC, and $\beta$-catenin. Furthermore, we correlated CDX2 protein expression with clinical data.

\section{Materials and methods}

Tissue samples. Formalin-fixed paraffin-embedded surgical specimens of liver metastasis of CRC were selected from the archives of the Institute of Pathology at the University Hospital of Heidelberg. Hundred and one patients without neo-adjuvant chemotherapy (64 male, 37 female; mean age 62 years) were included. Tumor size was between $5 \mathrm{~mm}$ and $16 \mathrm{~cm}$ in diameter. 12 cases showed mucinous adenocarcinoma histology and 89 cases showed histology of adenocarcinoma NOS. We had only two cases with grade 1 adenocarcinoma, 83 cases had grade 2 and 12 cases grade 3 histology. Serial paraffin sections were cut at $4 \mu \mathrm{m}$ for immunohistochemistry. Important clinical data, such as: Age, gender, size and number of metastases were collected from histological reports. Tissue samples were provided by the tissue bank of the National Centre for Tumor Diseases (NCT, Heidelberg, Germany) in accordance with the regulations of the tissue bank and the approval of the ethics committee of Heidelberg University according to ethical standards formulated in the Declaration of Helsinki 1975 (revised in 1983).

Tissue microarray (TMA). TMA blocks were punched from paraffin-embedded human liver specimens with a tissue microarrayer (Beecher Instruments, Sun Prairie, WI, USA). From each case, two cores of tumor tissue were punched with a diameter size of $1.6 \mathrm{~mm}$ and two muscle cores were used for orientation of the TMA slides. Therefore serial sections were cut from the TMA block. So far, there is no standardised operating protocol or universal agreement for sampling and staining of TMA blocks and slides. The general consensus is that at least two $0.6 \mathrm{~mm}$ cores adequately represent for immunohistochemical changes $(16,17)$.

Immunohistochemistry. $4 \mu \mathrm{m}$ thick slides were obtained from TMA blocks. Slides were then deparaffinised according to standard protocol by xylene, and dehydrated with $95-96 \%$ ethanol, 70\% ethanol and distilled water. All slides were stained simultaneously using a computer-controlled autostainer (Dako TechMate 500 cytomation) and Dako EnVision-Sytem (Dako; Agilent Technologies, Inc., Santa Clara, CA, USA) and pretreated with $3 \%$ Hydrogen Peroxide prior to antibody incubation. MLH1 [M1, ready-to-use (RTU), Ventana Medical Systems, Inc.; Roche Diagnostics, Basel, Switzerland], MSH6 (44, RTU; Ventana Medical Systems, Inc.; Roche Diagnostics), PMS2 (EPR3947, RTU; Ventana Medical Systems, Inc.; Roche Diagnostics), MSH2 (G219-1129, RTU; Ventana Medical Systems, Inc.; Roche Diagnostics), MGMT (MT-23.2; Thermo Fisher Scientific, Inc., Waltham, MA, USA; 1:20) and CDX2 (EPR2764Y; Thermo Fisher Scientific, Inc.; 1:200) antibodies were used. Secondary antibody binding (all Dako, 1:200) was 
visualised using a streptavidin ABC-kit (Dako), followed by 3,3'-diaminobenzidine (Vector, Peterborough, UK). For ERCC1 (8F1, Neomarkers; dilution: 1:100) and $\beta$-catenin (14, RTU; Ventana Medical Systems, Inc.; Roche Diagnostics) slides were stained by a computer-controlled autostainer (Ventana BenchMark Ultra; Ventana Medical Systems, Inc.; Roche Diagnostics). Polyclonal rabbit anti-APC antibody (DP2.5 1:200 Fa; Acris Antibodies; OriGene Technologies, Inc., Rockville, MD, USA) were used for APC staining. Staining was performed using ChemMate Detection kit (Dako) according to recommendations of the manufacturer. The antibodies were incubated overnight at $4{ }^{\circ} \mathrm{C}$ followed by avidin-biotin complex peroxidease technique using aminoethylcarbazole for visualization and hematoxylin for nuclear counterstaining. All slides were covered with Aquatex (Merck KGaA, Darmstadt, Germany).

Evaluation of immunohistochemistry. For the semi-quantitative assessment of staining intensity, we adjusted a previously published scoring system for each protein and fitted to TMA dots. For MSI proteins and for MGMT the staining was evaluated according to Bethesda guidelines (18): score 1, more than $10 \%$ of tumor cell nuclei are positive; score 0 , less than $10 \%$ of tumor cell nuclei positive (but: positive internal control, i.e., stromal cells and lymphocytes). The immunostained TMA sections were evaluated and scored under a light microscope independently by two pathologists in a blinded fashion. Discordant cases were reviewed and re-evaluated based on a consensus opinion.

Immunostaining for CDX2, ERCC1 and nuclear $\beta$-catenin was scored in a three-graded scale: score 0 , weak staining in less than $10 \%$ of the tumor cells; score 1 , moderate staining in up to $75 \%$ of the tumor cells; and score 2, strong nuclear staining in more than $75 \%$ of the tumor cells. For cytoplasmic $\beta$-catenin staining a two-graded scale was used: score 0 , no or weak staining in less than $10 \%$ of tumor cells and weaker staining compared to normal colonic mucosa; score 1, nuclear staining in more than $10 \%$ of the tumor cells.

Cytoplasmic and nuclear APC staining was separately scored. For nuclear APC staining a two-graded scale was used: score 0 , No or weaker staining in less than $10 \%$ of tumor cells and weaker staining compared to normal colonic mucosa; score 1 , nuclear staining in more than $10 \%$ of the tumor cells. For cytoplasmic APC staining a three-graded scale was used: score 0 , no cytoplasmic staining or weak staining in less than $10 \%$ of tumor cells; score $1,10-75 \%$ of the tumor cells with moderate intensity; and score 2 , more than $75 \%$ of the tumor cells are positive with high staining intensity. Normal colorectal mucosa was set as baseline expression level for APC (score 2).

Statistical analysis. The statistical analyses were performed with SAS software (SAS Institute, Inc., Cary, NC, USA). Spearman-Rho test was used to evaluate the relationship between clinical data, CDX2, MLH1, MSH2, MSH6, PMS2, MGMT, ERCC1, APC and $\beta$-catenin.

\section{Results}

CDX2 expression and its correlation with clinical data. We could reach valid expression data for CDX2 (Table I) in 83 of 101 cases. 32 cases $(38.55 \%)$ show no nuclear expression.
Positive stainings $(61.45 \%, \mathrm{n}=51 / 83)$ can be subdivided into two groups: Moderate nuclear expression with score $1(16.87 \%$ $\mathrm{n}=14)$; and strong positivity with score $2(44.58 \% \%, \mathrm{n}=37)$. Representative photomicrographs of CDX2 immunohistochemistry are depicted in Fig. 1.

Concerning clinical parameters like: Age, gender of the patients, grading of the tumor and the number of metastases, there was no significant correlation to CDX2 expression. Regarding the size of the metastasis a strong negative correlation could be detected ( $\mathrm{P}=0.038)$. In addition to $\mathrm{CDX} 2$, ERCC1 expression was also strongly correlated with the size of the metastases $(\mathrm{P}=0.027)$. Bigger metastasis size diameter was seen in cases with CDX2 and ERCC1 loss.

Expression distribution of DNA repair proteins and proteins involved in Wnt-signaling. For MGMT 97 valid cases were obtained. Loss of MGMT expression was found in 24 cases (24.75\%). Representative photomicrographs of MGMT immunohistochemistry are depicted in Fig. 2. Nuclear positivity was sustained in 73 cases $(75.25 \%)$. Out of 94 valid cases for ERCC1 we found $29.8 \%$ of the cases negative (score 0 ). Positive ERCC1 staining could be in $70.2 \%$ of the cases detected $(30.8 \%$ score 1 and $39.4 \%$ score 2). Representative photomicrographs of ERCC1 immunohistochemistry are depicted in Fig. 3. Both MGMT and ERCC1 loss is strongly associated with female gender ( $\mathrm{P}=0.011$, and $\mathrm{P}=0.047$, respectively).

Regarding MMR proteins, the following distribution was seen: Loss of expression was detected in 4.2 to $26 \%$ of the cases (MLH1 4.2\%, MSH2 26\%, MSH6 24\% and PMS2 $9.5 \%$, respectively) as published before (19). Loss of PMS2 is associated with loss of MGMT $(\mathrm{P}=0.014)$ and loss of MLH1 and $\mathrm{MSH} 2$ were also associated with loss of ERCC1 $(\mathrm{P}<0.01$, and $\mathrm{P}<0.01$, respectively). Representative photomicrographs of MMR protein immunohistochemistry are depicted in Fig. 4. Expression distribution of $\beta$-catenin, and APC proteins are depicted in Table I.

Statistical correlations between CDX2 and DNA repair proteins. We found statistically strong positive correlation between CDX2 and all of analysed DNA repair proteins (Table II). These results mean that loss of CDX2 expression is strongly associated with loss of expression of DNA repair proteins (MMR proteins, MGMT and ERCC1).

Statistical correlations between CDX2, APC and $\beta$-catenin. We analysed the possible statistical correlation between CDX2 and $\beta$-catenin, and APC (Table III). Cytoplasmic, but not nuclear $\beta$-catenin expression is associated with sustained nuclear CDX2 expression ( $\mathrm{P}=0.042)$. In addition, CDX2 is positively correlated with nuclear APC expression $(\mathrm{P}<0.01)$. Cytoplasmic and nuclear $\beta$-catenin is associated also positive with each other $(\mathrm{P}<0.01)$. Representative photomicrographs of APC and $\beta$-catenin immunohistochemistry are depicted in Figs. 5 and 6, respectively.

\section{Discussion}

In this study, we have demonstrated significant correlations between CDX2, DNA repair proteins and crucial members of Wnt signaling. To our knowledge, this is the first report 
Table I. Distribution of immunostaining results of CDX2, APC and $\beta$-catenin.

\begin{tabular}{lcccc}
\hline Protein & Score 0 $(\%)$ & Score 1 $(\%)$ & Score 2 $(\%)$ & No. of valid cases $(\%)$ \\
\hline CDX2 & $32(38.55)$ & $14(16.87)$ & $37(44.58)$ & $83(100)$ \\
Nuclear APC & $62(61.38)$ & $39(38.62)$ & - & $101(100)$ \\
Cytoplasmic APC & $13(12.87)$ & $75(74.26)$ & $13(12.87)$ & $101(100)$ \\
Cytoplasmic $\beta$-catenin & $37(38.14)$ & $60(61.86)$ & - & $97(100)$ \\
Nuclear $\beta$-catenin & $60(61.86)$ & $21(21.65)$ & $16(16.49)$ & $97(100)$
\end{tabular}

CDX2, caudal type homeobox 2; APC, adenomatous polyposis coli.
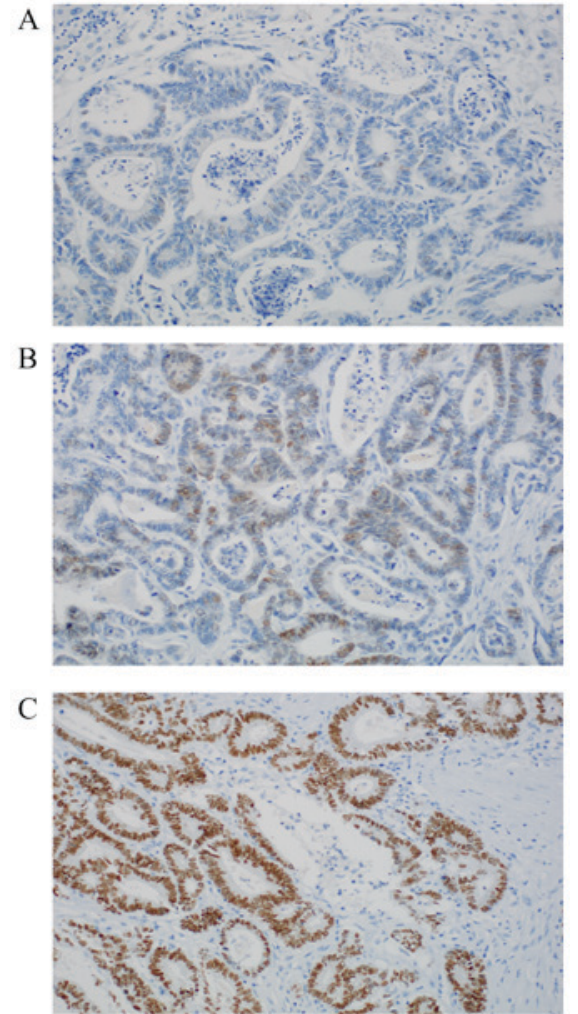

Figure 1. Representative examples of caudal type homeobox 2 protein expression in colorectal liver metastasis. (A) Score 0, negative staining$<10 \%$ of the nuclei are stained. (B) Score 1, moderate overexpression in $<75 \%$ of the nuclei. (C) Score 2, strong overexpression in almost all tumor cells. Magnification, x200.

performed on human tissue of CRC liver metastasis presenting statistically significant correlations between expression of CDX2 referring to expression of MMR proteins and key proteins of base and nuclear excision repair. Furthermore, we show, for the first time, significant correlation between CDX2, APC and $\beta$-catenin in liver metastasis of CRC.

Loss of CDX2 expression is seen in approximately $30 \%$ of human CRC and is associated with higher tumor grade (1). We found loss of CDX2 expression in $38.55 \%$ of the cases. Loss of CDX2 expression was negatively correlated with tumor size, but no correlation with age, gender of the patients, grade of the tumor and the number of metastases. Interestingly, ERCC1 expression loss was also correlated with tumor size. Furthermore, loss of CDX2 is strongly correlated with loss of

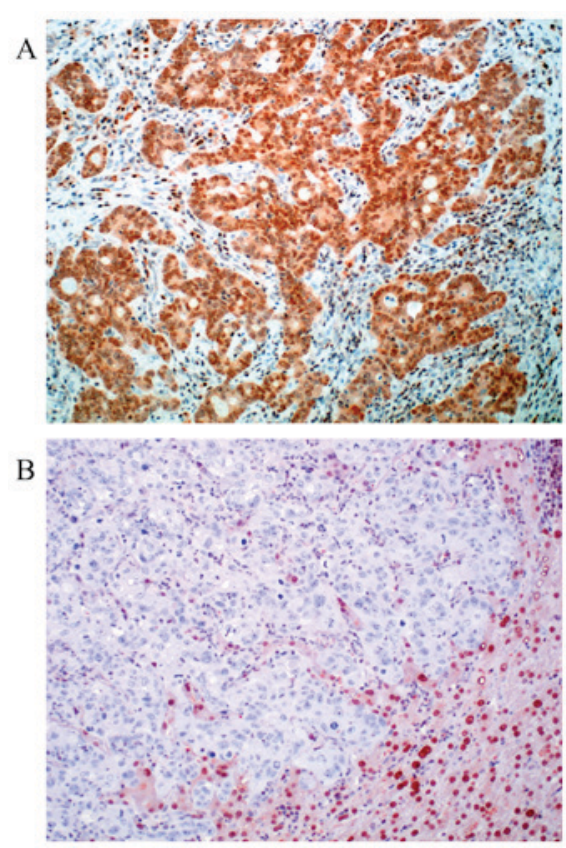

Figure 2. Representative examples of O6-methylguanine DNA methyltransferase protein expression in colorectal liver metastasis. (A) Score 0, negative staining- $<10 \%$ of the nuclei are stained. (B) Score $1,>10 \%$ percent of the nuclei are positive. Note that in negative cases, stromal cells, lymphocytes and hepatocytes are still nuclear positive. Magnification, x200.

ERCC1. Thus, we can conclude, that loss of CDX2 or ERCC1 expression is strongly associated with bigger metastatic tumor size. Similar results for ERCC1 were found recently in breast cancer (20), but the exact mechanisms are still unclear.

We can demonstrate statistically significant correlations between CDX2 and DNA repair proteins: Loss of CDX2 expression is associated with loss of MMR proteins, MGMT, and ERCC1. These results are consistent with literature data from primary CRC: MMR-deficient or MSI high CRCs have significant losses of CDX2 expression. In addition, loss of CDX2 is associated with CIMP-high, more aggressive histomorphological features, and unfavourable survival (21). In a study on primary $\mathrm{CRC}$ and its lymph node metastasis reduced expression of CDX2 were found to be as predictor of MMR-deficiency in CRC. Moreover, loss of CDX2 is a poor prognostic factor, even among patients with MMR-proficient cancers (22).

Mutations in DNA repair genes are rare in sporadic cancers with DNA repair deficiency. However, DNA repair deficiency occurs in a majority of sporadic cancers caused 


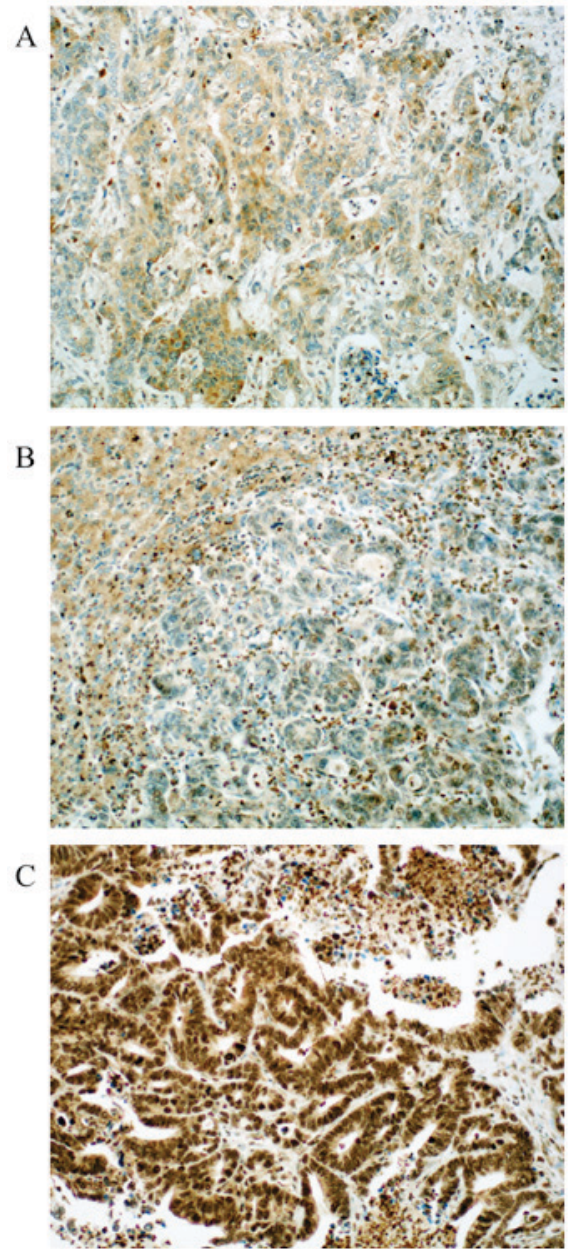

Figure 3. Representative examples of excision repair cross-complementing 1 protein expression in colorectal liver metastasis. (A) Score 0, no expression detectable or faint partial expression in $<10 \%$ of the tumor cells. (B) Score 1 , weak to moderate expression of the entire tumor tissue. (C) Score 2, strong positivity in the entire tumor tissue. Magnification, x200.

by epigenetic alterations that reduce or silence DNA repair gene expression. For example, a majority of primary CRCs have reduced MGMT expression due to i.e., methylation of the MGMT promoter region (an epigenetic alteration) (23). MGMT can be epigenetically depressed in many ways. Beside hypermethylation, MGMT can be depressed by di-methylation of lysine 9 of histone 3 (24) or by over-expression of a number of microRNAs including miR-181d, miR-767-3p and miR-603 (25).

Methylation of MGMT promoter region plays a significant role not only in carcinogenesis but also predictive for therapy response. In glioblastoma multiforme, the methylation state of the MGMT gene determined whether patients would be responsive to temozolomide therapy (26). On a clinical level, this translates into a prolonged survival of glioblastoma patients with a methylated MGMT promoter. In addition, MGMT methylation can be used to predict patient survival in clinical prediction models (27).

Loss of MGMT and ERCC1 expression was associated with female sex in our study. Similar data were demonstrated in primary CRC for MGMT (28) and for ERCC1 in lung cancer (29), thus we can conclude that this phenomenon stay maintained in liver metastasis. For ERCC1 our study is the first

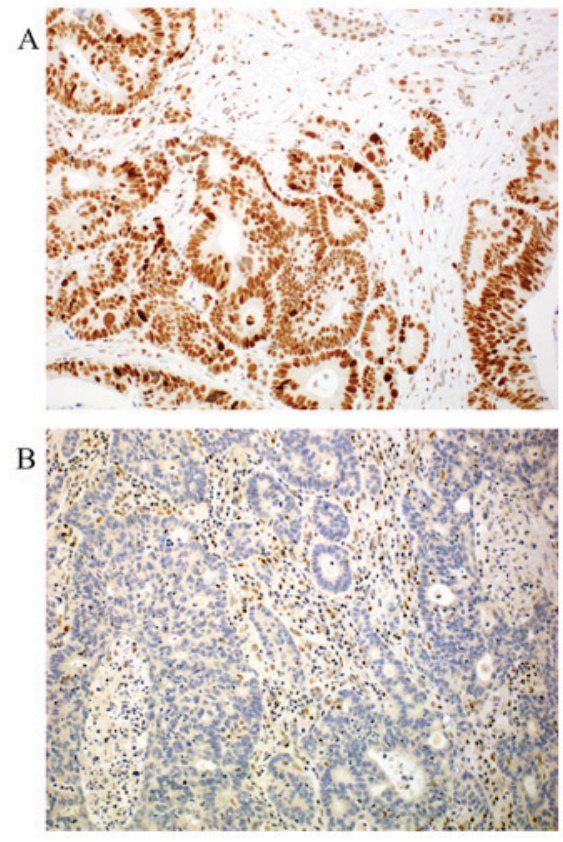

Figure 4. Representative examples of mismatch repair system protein expression (MutL Homolog 1 staining) in colorectal liver metastasis. (A) Score 0, negative staining- $<10 \%$ of the nuclei are stained. (B) Score $1,>10 \%$ percent of the nuclei are positive. Note that in negative cases, stromal cells are still positive. Magnification, x200.

demonstrating statistically significant correlation with female gender in CRC. ERCC1 is essential for a functional NER system and ERCC1 expression loss may contribute to impaired DNA repair capacity thus increasing cancer risk. Reduced expression or loss of ERCC1 and MGMT were reported in vast majority of CRCs $(30,31)$, and ERCC1 promoter hypermethylation in $38 \%$ of gliomas, resulting in reduced mRNA and protein expression (32). Disturbed ERCC1 protein expression appears to be an early event in colorectal carcinogenesis: reduced or loss of ERCC1 expression was detected in $40 \%$ of the colonic crypts within early field defects in colorectal mucosa (30). Similarly to MGMT, ERCC1 silencing can be resulted not only from promoter methylation, but can also be evocated by miRNAs repressing its expression (33). Whether epigenetic mechanisms reduce ERCC1 and MGMT protein expression in liver metastasis of $\mathrm{CRC}$ has to be determined in methylation studies. In general, the exact role of ERCC1 should be further elucidated because of its predictive role in chemotherapy. Pre-clinical studies have demonstrated its important role in determining cisplatin resistance (34).

In summary, loss of CDX2 is associated with each DNA repair protein, which we analysed and our results in liver metastasis are in accordance with the literature data originated from primary CRC $(21,22)$. Loss of CDX2 has also been found to be an independent predictor of the CIMP-high phenotype (22). We used MGMT as surrogate marker for CIMP phenotype, but it has been noted that studies about MGMT methylation and CIMP had inconsistent findings, thus tumors with loss of MGMT cannot be clearly classified as CIMP phenotype (35). CIMP-high CRCs have been reported to have a different clinicopathological features than CIMP-low ones. CIMP-high phenotype is associated with older age, cigarette smoking, proximal tumor location, female gender, poorly differentiated 
Table II. Results of statistical analysis between CDX2, DNA repair proteins and tumor size.

DNA repair proteins

\begin{tabular}{lcccccccc}
\cline { 4 - 8 } Gene & Analysis & Tumor size $(\mathrm{mm})$ & MLH1 & MSH2 & MSH6 & PMS2 & MGMT & ERCC1 \\
\hline CDX2 & Correlation coefficient & $-0.247^{\mathrm{a}}$ & $0.388^{\mathrm{b}}$ & $0.334^{\mathrm{b}}$ & $0.317^{\mathrm{b}}$ & $0.228^{\mathrm{a}}$ & $0.236^{\mathrm{a}}$ & $0.574^{\mathrm{b}}$ \\
& Significance (2-sided) & 0.038 & $<0.001$ & 0.002 & 0.004 & 0.040 & 0.039 & $<0.001$ \\
& Number of valid cases & 71 & 77 & 82 & 82 & 82 & 77 & 74 \\
\hline
\end{tabular}

${ }^{\mathrm{a}} \mathrm{P}<0.05$ and ${ }^{\mathrm{b}} \mathrm{P}<0.01$. CDX2, caudal type homeobox 2; MLH1, MutL Homolog 1; MSH, MutS protein Homolog; PMS2, postmeiotic segregation increased 2; MGMT, O6-methylguanine DNA methyltransferase; ERCC1, excision repair cross-complementing 1.

Table III. Results of statistical analysis between CDX2, APC and $\beta$-catenin.

\begin{tabular}{lccccc}
\hline Gene & Analysis & $\begin{array}{c}\text { Membranous/cytoplasmic } \\
\beta \text {-catenin }\end{array}$ & Nuclear $\beta$-catenin & Cytoplasmic APC & Nuclear APC \\
\hline CDX2 & Correlation coefficient & $0.231^{\text {a }}$ & 0.152 & 0.065 & $0.415^{\text {b }}$ \\
& Significance (2-sided) & 0.042 & 0.183 & 0.567 & $<0.001$ \\
& Number of valid cases & 78 & 78 & 79 & 79 \\
\hline
\end{tabular}

${ }^{\mathrm{a}} \mathrm{P}<0.05$ and ${ }^{\mathrm{b}} \mathrm{P}<0.01$. CDX2, caudal type homeobox 2 ; APC, adenomatous polyposis coli.

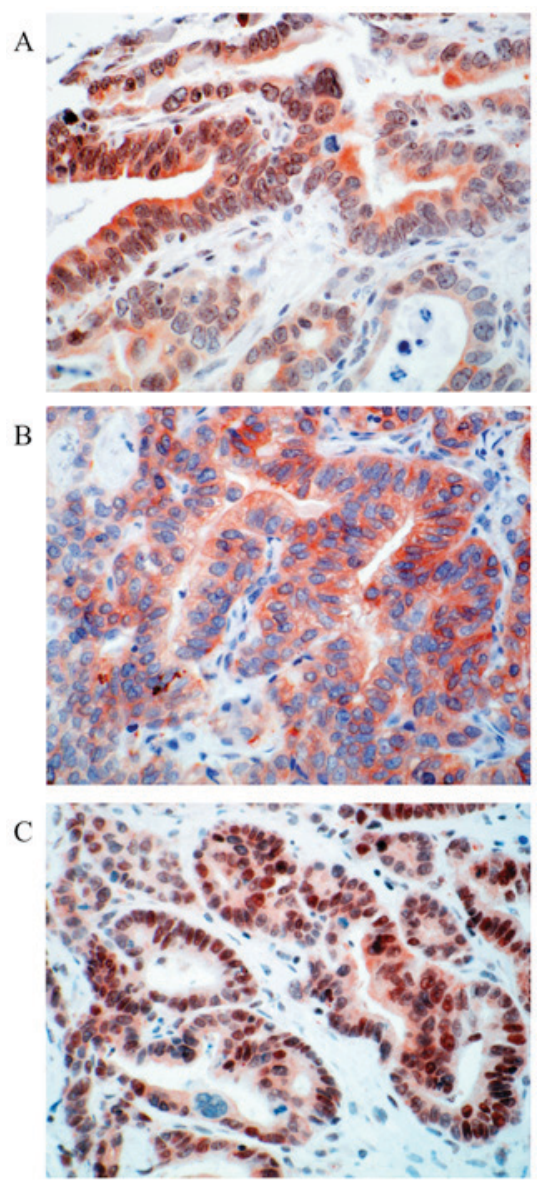

Figure 5. Representative examples of APC protein expression in colorectal liver metastasis. (A) Cytoplasmic and nuclear expression of APC protein (B) Only cytoplasmic APC positivity in the tumor cells was observed. (C) Strong nuclear positivity next to faint cytoplasmic staining. Magnification, $\mathrm{x} 400$. APC, adenomatous polyposis coli.

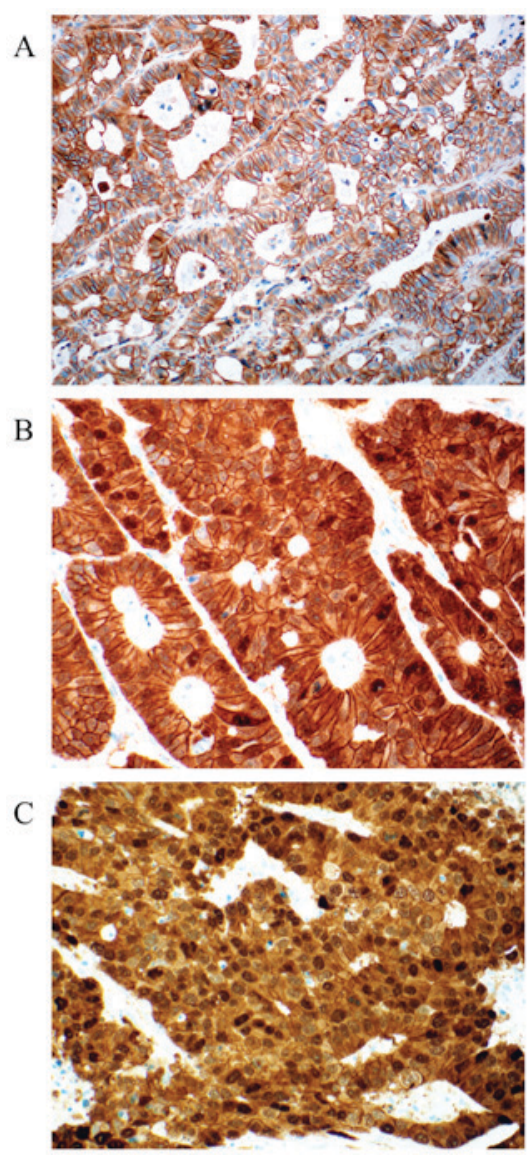

Figure 6. Representative examples of $\beta$-catenin expression in colorectal liver metastasis. (A) Only membranous staining was observed (score 0 for cytoplasm and nucleus). (B) Cytoplasmic expression of $\beta$-catenin with some positive nuclei (score 1 for cytoplasm and nuclei). (C) Diffuse and strong cytoplasmic and nuclear staining (score 1 for cytoplasm, and score 2 for nuclei). Magnification, x200. 
or mucinous adenocarcinoma, MSI, and BRAF mutation. In addition, CIMP-high cancers regardless of microsatellite status show a poorer outcome (36). We suggest that MGMT is an adequate marker to detect CIMP phenotype.

The Wnt $\beta \beta$-catenin pathway is a crucial signalling pathway in control of embryonic development and tissue homeostasis. Its deregulation is observed in many cancers (i.e., CRC, non-hepatitis-related hepatocellular cancers, cholangiocarcinoma, desmoid tumor, breast cancer, osteosarcoma etc) (37). The pathway is over-activated in almost all colon cancer because of mutations of APC tumor suppressor gene, which actually represent the initiating event in colorectal carcinogenesis (38). Nevertheless, the actual mechanisms, which regulate $\beta$-catenin still remain highly controversial. Furthermore, the exact role of APC in particular is unclear, and the consequences of the mutations found in cancer cells are still poorly defined (38). Subcellular localisation of APC protein is differentially regulated in normal tissues and cell lines: in normal human colorectal epithelium, APC is located in the nuclei at basal segment of the crypts; in HT29 colon cancer cells, truncated APC translocated to the nucleus during early apoptosis (39), and cellular APC accumulates in the nucleus of sub-confluent cells but is partly excluded in super-confluent cells (14). Although there is consensus in many areas in the field of nuclear APC localization and function, there have also been some conflicting results with no apparent resolution. Moreover, the specificity of several APC antibodies has been investigated, with no clear consensus about the 'best' antibody to detect APC protein (40). The nuclear transport of APC in tumor cells occurs independently of $\beta$-catenin translocation to the nucleus or plasma membrane (41).

Nuclear accumulation of $\beta$-catenin is also observed in cancers resulting from mutations in the $\beta$-catenin, APC or Axin genes $(15,42)$. The APC tumor suppressor binds to $\beta$-catenin and the scaffold protein Axin to form a complex promoting GSK-3 $\beta$ phosphorylation of $\beta$-catenin. However, overexpression of APC (1-1309), the most frequently occurring APC cancer mutant, translocates $\beta$-catenin from the nucleus to the cytoplasm. This mutant therefore has the ability to bind and regulate localization but lacks the Axin binding sites required for $\beta$-catenin degradation. Therefore, it seems more likely that it is the inability of APC to promote $\beta$-catenin degradation, rather than a lack of export function, that causes the nuclear accumulation of $\beta$-catenin in APC-mutant tumor cells (14).

Little is known about the connections between CDX2 and Wnt signaling pathway. In a study on Caco-2 cells lower CDX2 expression is associated with endogenous downregulation of APC expression, but did not affect GSK3 $\beta$ expression (4). Our analysis led to similar results: Reduced expression or loss of CDX2 is associated with reduced nuclear APC expression $(\mathrm{P}<0.01)$. In our study, the cytoplasmic APC expression was not associated with CDX2 expression. We assume that although CDX2 induce APC expression, which is already proven (4), the truncated APC protein cannot be shifted to cytoplasm, but we could detect this truncated protein with our antibody. In conclusion, truncated APC can be detected with immunohistochemistry and has certainly not lost its full function and can still participate in $\beta$-catenin regulation. Thus, APC can still fulfil an unexpectedly large spectrum of APC function (38). Furthermore, we found statistically significant correlation between CDX2 and cytoplasmic $\beta$-catenin. We think this correlation can be explained through the Mucdhl, a common interaction partner for $\beta$-catenin and CDX2. It has been shown that $\beta$-catenin interacts with a protocadherin Mucdhl, which is regulated by CDX2 in mice. Membrane-bound $\beta$-catenin is a consequence of interactions to membranous-expressed Mucdhl. Thus, Mucdhl can inhibit $\beta$-catenin translocation to the nucleus (4).

CDX2 is indeed expressed in all stages of CRC, little is known about its expression manner in association with other established prognostic or predictive proteins. In this report, we have directly demonstrated that CDX2 gene expression is strongly associated with DNA repair proteins and crucial members of Wnt signaling. Our results further strengthen the role of CDX2 in DNA repair and in regulation of APC and $\beta$-catenin expression. In fact, our analysis is restricted only for metastasis, our results strongly suggest potential (functional) interactions between the investigated proteins. To our knowledge, this is the first study to investigate CDX2 in this context on human liver metastasis of CRC. Although, CDX2 is a useful marker in routine diagnostics for CRC, its exact role in liver metastasis remains to be further elucidated. In further studies should be investigated if primary CRC differs from liver metastasis regarding CDX2 expression.

\section{Acknowledgements}

This study was supported by GINOP 2.3.2-15-2016-00020 project, which is co-financed by the European Regional Developmental Fund of the European Union.

\section{References}

1. Hryniuk A, Grainger S, Savory JG and Lohnes D: Cdx1 and Cdx2 function as tumor suppressors. J Biol Chem 289: 33343-33354, 2014.

2. Misiakos EP, Karidis NP and Kouraklis G: Current treatment for colorectal liver metastases. World J Gastroenterol 17: 4067-4075, 2011.

3. Olsen J, Espersen ML, Jess P, Kirkeby LT and Troelsen JT: The clinical perspectives of CDX2 expression in colorectal cancer: A qualitative systematic review. Surg Oncol 23: 167-176, 2014.

4. Olsen AK, Coskun M, Bzorek M, Kristensen MH, Danielsen ET, Jørgensen S, Olsen J, Engel U, Holck S and Troelsen JT: Regulation of APC and AXIN2 expression by intestinal tumor suppressor CDX2 in colon cancer cells. Carcinogenesis 34: 1361-1369, 2013.

5. Zlobec I, Bihl M, Foerster A, Rufle A and Lugli A: Comprehensive analysis of $\mathrm{CpG}$ island methylator phenotype (CIMP)-high, -low and -negative colorectal cancers based on protein marker expression and molecular features. J Pathol 225: 336-343, 2011.

6. Renouf B, Soret C, Saandi T, Delalande F, Martin E, Vanier M, Duluc I, Gross I, Freund JN and Domon-Dell C: Cdx2 homeoprotein inhibits non-homologous end joining in colon cancer but not in leukemia cells. Nucleic Acids Res 40: 3456-3469, 2012.

7. Li X, Hu F, Wang Y, Yao X, Zhang Z, Wang F, Sun G, Cui BB Dong $\mathrm{X}$ and Zhao Y: $\mathrm{CpG}$ island methylator phenotype and prognosis of colorectal cancer in Northeast China. Biomed Res Int 2014: 236361, 2014.

8. Inno A, Fanetti G, Di Bartolomeo M, Gori S, Maggi C, Cirillo M, Iacovelli R, Nichetti F, Martinetti A and de Braud F: Role of MGMT as biomarker in colorectal cancer. World J Clin Cases 2: 835-839, 2014.

9. Hassen S, Ali N and Chowdhury P: Molecular signaling mechanisms of apoptosis in hereditary non-polyposis colorectal cancer. World J Gastrointest Pathophysiol 3: 71-79, 2012

10. Sayar I, Akbas EM, Isik A, Gokce A, Peker K, Demirtas L and Gürbüzel M: Relationship among mismatch repair deficiency, CDX2 loss, p53 and E-cadherin in colon carcinoma and suitability of using a double panel of mismatch repair proteins by immunohistochemistry. Pol J Pathol 66: 246-253, 2015. 
11. Li Z, Qing Y, Guan W, Li M, Peng Y, Zhang S, Xiong Y and Wang D: Predictive value of APE1, BRCA1, ERCC1 and TUBB3 expression in patients with advanced non-small cell lung cancer (NSCLC) receiving first-line platinum-paclitaxel chemotherapy. Cancer Chemother Pharmacol 74: 777-786, 2014.

12. Ruzzo A, Graziano F, Loupakis F, Rulli E, Canestrari E, Santini D, Catalano V, Ficarelli R, Maltese P, Bisonni R, et al: Pharmacogenetic profiling in patients with advanced colorectal cancer treated with first-line FOLFOX-4 chemotherapy. J Clin Oncol 25: 1247-1254, 2007.

13. Persad S, Troussard AA, McPhee TR, Mulholland DJ and Dedhar S: Tumor suppressor PTEN inhibits nuclear accumulation of beta-catenin and T cell/lymphoid enhancer factor 1-mediated transcriptional activation. J Cell Biol 153: 1161-1174, 2001.

14. Henderson BR and Fagotto F: The ins and outs of APC and beta-catenin nuclear transport. EMBO Rep 3: 834-839, 2002

15. Polakis P: Wnt signaling and cancer. Genes Dev 14: 1837-1851, 2000.

16. Camp RL, Charette LA and Rimm DL: Validation of tissue microarray technology in breast carcinoma. Lab Invest 80 : 1943-1949, 2000.

17. Torhorst J, Bucher C, Kononen J, Haas P, Zuber M, Köchli OR, Mross F, Dieterich H, Moch H, Mihatsch M, et al: Tissue microarrays for rapid linking of molecular changes to clinical endpoints. Am J Pathol 159: 2249-2256, 2001.

18. Umar A, Boland CR, Terdiman JP, Syngal S, de la Chapelle A, Rüschoff J, Fishel R, Lindor NM, Burgart LJ, Hamelin R, et al: Revised bethesda guidelines for hereditary nonpolyposis colorectal cancer (Lynch syndrome) and microsatellite instability. J Natl Cancer Inst 96: 261-268, 2004.

19. Toth C, Meinrath J, Herpel E, Derix J, Fries J, Buettner R, Schirmacher $\mathrm{P}$ and Heikaus S: Expression of the apoptosis repressor with caspase recruitment domain (ARC) in liver metastasis of colorectal cancer and its correlation with DNA mismatch repair proteins and p53. J Cancer Res Clin Oncol 142: 927-935, 2015.

20. Gerhard R, Carvalho A, Carneiro V, Bento RS, Uemura G, Gomes M, Albergaria A and Schmitt F: Clinicopathological significance of ERCC1 expression in breast cancer. Pathol Res Pract 209: 331-336, 2013.

21. Dawson H,Galvan JA, Helbling M, MullerDE,Karamitopoulou E, Koelzer VH, Economou M, Hammer C, Lugli A and Zlobec I: Possible role of $\mathrm{Cdx} 2$ in the serrated pathway of colorectal cancer characterized by BRAF mutation, high-level CpG Island methylator phenotype and mismatch repair-deficiency. Int J Cancer 134: 2342-2351, 2014

22. Dawson H, Koelzer VH, Lukesch AC, Mallaev M, Inderbitzin D, Lugli A and Zlobec I: Loss of $\mathrm{Cdx} 2$ expression in primary tumors and lymph node metastases is specific for mismatch repair-deficiency in colorectal cancer. Front Oncol 3: 265, 2013.

23. Halford S, Rowan A, Sawyer E, Talbot I and Tomlinson I: $\mathrm{O}(6)$-methylguanine methyltransferase in colorectal cancers: Detection of mutations, loss of expression and weak association with G:C>A:T transitions. Gut 54: 797-802, 2005.

24. Nakagawachi T, Soejima H, Urano T, Zhao W, Higashimoto K, Satoh Y, Matsukura S, Kudo S, Kitajima Y, Harada H, et al: Silencing effect of $\mathrm{CpG}$ island hypermethylation and histone modifications on O6-methylguanine-DNA methyltransferase (MGMT) gene expression in human cancer. Oncogene 22: 8835-8844, 2003

25. Cabrini G, Fabbri E, Lo Nigro C, Dechecchi MC and Gambari R: Regulation of expression of O6-methylguanine-DNA methyltransferase and the treatment of glioblastoma (Review). Int $\mathrm{J}$ Oncol 47: 417-428, 2015

26. Hegi ME, Diserens AC, Gorlia T, Hamou MF, de Tribolet N, Weller M, Kros JM, Hainfellner JA, Mason W, Mariani L, et al: MGMT gene silencing and benefit from temozolomide in glioblastoma. N Engl J Med 352: 997-1003, 2005.
27. Molenaar RJ, Verbaan D, Lamba S, Zanon C, Jeuken JW, Boots-Sprenger SH, Wesseling P, Hulsebos TJ, Troost D, van Tilborg AA, et al: The combination of IDH1 mutations and MGMT methylation status predicts survival in glioblastoma better than either IDH1 or MGMT alone. Neuro Oncol 16: 1263-1273, 2014.

28. Shen L, Kondo Y, Rosner GL, Xiao L, Hernandez NS, Vilaythong J, Houlihan PS, Krouse RS, Prasad AR, Einspahr JG, et al: MGMT promoter methylation and field defect in sporadic colorectal cancer. J Natl Cancer Inst 97: 1330-1338, 2005.

29. Kalogeraki A, Karvela-Kalogeraki I, Tamiolakis D, Petraki P, Saridaki Z and Tzardi M: ERCC1 expression correlated with EGFR and clinicopathological variables in patients with non-small cell lung cancer. An immunocytochemical study on fine-needle aspiration biopsies samples. Rev Port Pneumol 20: 200-207, 2014.

30. Facista A, Nguyen H, Lewis C, Prasad AR, Ramsey L, Zaitlin B, Nfonsam V, Krouse RS, Bernstein H, Payne CM, et al: Deficient expression of DNA repair enzymes in early progression to sporadic colon cancer. Genome Integr 3: 3, 2012.

31. Smith DH, Fiehn AM, Fogh L, Christensen IJ, Hansen TP, Stenvang J, Nielsen HJ, Nielsen KV, Hasselby JP, Brünner N and Jensen SS: Measuring ERCC1 protein expression in cancer specimens: Validation of a novel antibody. Sci Rep 4: 4313, 2014.

32. Chen HY, Shao CJ, Chen FR, Kwan AL and Chen ZP: Role of ERCC1 promoter hypermethylation in drug resistance to cisplatin in human gliomas. Int J Cancer 126: 1944-1954, 2010

33. Borrmann L, Schwanbeck R, Heyduk T, Seebeck B, Rogalla P, Bullerdiek J and Wisniewski JR: High mobility group A2 protein and its derivatives bind a specific region of the promoter of DNA repair gene ERCC1 and modulate its activity. Nucleic Acids Res 31: 6841-6851, 2003.

34. Garofalo M and Croce CM: MicroRNAs as therapeutic targets in chemoresistance. Drug Resist Updat 16: 47-59, 2013.

35. Hawkins NJ, Lee JH, Wong JJ, Kwok CT, Ward RL and Hitchins MP: MGMT methylation is associated primarily with the germline C>T SNP (rs16906252) in colorectal cancer and normal colonic mucosa. Mod Pathol 22: 1588-1599, 2009.

36. Kang KJ, Min BH, Ryu KJ, Kim KM, Chang DK, Kim JJ, Rhee JC and Kim YH: The role of the $\mathrm{CpG}$ island methylator phenotype on survival outcome in colon cancer. Gut Liver 9: 202-207, 2015.

37. Pai SG, Carneiro BA, Mota JM, Costa R, Leite CA, Barroso-Sousa R, Kaplan JB, Chae YK and Giles FJ: Wnt/beta-catenin pathway: Modulating anticancer immune response. J Hematol Oncol 10: 101, 2017.

38. Wang L, Liu X, Gusev E, Wang C and Fagotto F: Regulation of the phosphorylation and nuclear import and export of $\beta$-catenin by APC and its cancer-related truncated form. J Cell Sci 127: 1647-1659, 2014.

39. Efstathiou JA, Noda M, Rowan A, Dixon C, Chinery R, Jawhari A, Hattori T, Wright NA, Bodmer WF and Pignatelli M: Intestinal trefoil factor controls the expression of the adenomatous polyposis coli-catenin and the E-cadherin-catenin complexes in human colon carcinoma cells. Proc Natl Acad Sci USA 95: 3122-3127, 1998

40. Neufeld KL: Nuclear APC. Adv Exp Med Biol 656: 13-29, 2009.

41. Fagman H, Larsson F, Arvidsson Y, Meuller J, Nordling M, Martinsson T, Helmbrecht K, Brabant $G$ and Nilsson M: Nuclear accumulation of full-length and truncated adenomatous polyposis coli protein in tumor cells depends on proliferation. Oncogene 22: 6013-6022, 2003.

42. Fodde R, Smits R and Clevers H: APC, signal transduction and genetic instability in colorectal cancer. Nat Rev Cancer 1: 55-67, 2001. 